\title{
Comparison of Free Radical Levels in the Aerosol from Conventional Cigarettes, Electronic Cigarettes, and Heat-Not-Burn Tobacco Products
}

\author{
Mikhail Shein ${ }^{\dagger}$ and Gunnar Jeschke*(i) \\ Department of Chemistry and Applied Biosciences, ETH Zürich, Vladimir-Prelog-Weg 2, 8093 Zurich, Switzerland
}

Supporting Information

\begin{abstract}
Aerosols from electronic cigarettes and heat-not-burn tobacco products have been found to contain lower levels of almost all compounds from the list of Harmful and Potentially Harmful Constituents known to be present in tobacco products and tobacco smoke than smoke from conventional cigarettes. Free radicals, which also pose potential health risks, are not considered in this list, and their levels in the different product types have not yet been compared under standardized conditions. We compared the type and quantity of free radicals in mainstream aerosol of 3R4F research cigarettes, two types of electronic cigarettes, and a heat-not-burn tobacco product. Free radicals and NO in the gas phases

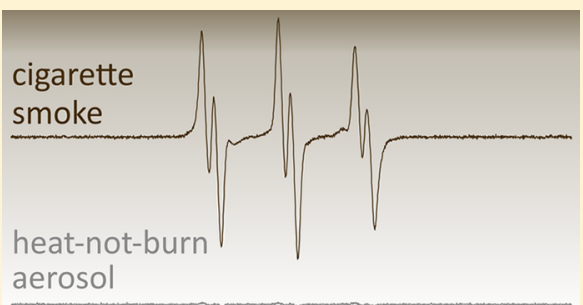
were separately spin trapped and quantified by electron paramagnetic resonance (EPR) spectroscopy by using a smoking machine for aerosol generation and a flow-through cell to enhance reproducibility of the quantification. Particulate matter was separated by a Cambridge filter and extracted, and persistent radicals were quantified by EPR spectroscopy. Levels of organic radicals for electronic cigarettes and the heat-not-burn product, as measured with the PBN spin trap, did not exceed $1 \%$ of the level observed for conventional cigarettes and were close to the radical level observed in air blanks. The radicals found in the smoke of conventional cigarettes were oxygen centered, most probably alkoxy radicals, whereas a signal for carbon-centered radicals near the detection limit was observed in aerosol from the heat-not-burn product and electronic cigarettes. The NO level in aerosol produced by electronic cigarettes was below our detection limit, whereas for the heat-not-burn product, it reached about $7 \%$ of the level observed for whole smoke from 3R4F cigarettes. Persistent radicals in particulate matter could be quantified only for $3 \mathrm{R} 4 \mathrm{~F}$ cigarettes. Aerosols from vaping and heat-not-burn tobacco products have much lower free radical levels than cigarette smoke, however, the toxicological implications of this finding are as yet unknown.
\end{abstract}

\section{INTRODUCTION}

Tobacco is widely consumed as a stimulant, mostly by inhalation of the smoke of cigarettes. Cigarette smoke contains a number of carcinogenic, mutagenic, or otherwise toxic substances as well as the addictive substance nicotine. It is recognized by the World Health Organization (WHO) as a major health hazard and contributor to mortality, ${ }^{1}$ and, as a basis for comparing the risk of different products, a list of 93 Harmful and Potentially Harmful Constituents (HPHCs) in Tobacco Products and Tobacco Smoke has been published by the U.S. Food and Drug Administration (FDA). ${ }^{2}$ The health risk combined with the addictive nature of tobacco has led to a search for less harmful alternatives, ${ }^{3,4}$ such as electronic cigarettes (e-cigs) and heat-not-burn (HNB) tobacco products. While there is evidence for a reduced risk ${ }^{4,5}$ and a recent report of the National Academies of Sciences, Engineering, and Medicine suggests that use of e-cigs may have a net health benefit if it leads to widespread adult cessation of combustible tobacco products, ${ }^{6}$ toxicological effects of e-cig aerosol inhalation observed in a rat lung model suggest that a cancer risk still persists. ${ }^{7}$ Levels of some HPHCs selected from the FDA list have been found to be reduced by a factor of 5-100 in heat-not-burn products as compared to conventional cigarettes. ${ }^{8}$ However, the FDA list does not cover all classes of HPHCs. Free radicals have been found in cigarette smoke as early as $1958^{9}$ and were implicated in DNA damage ${ }^{10}$ and cardiovascular disease. ${ }^{11}$ The reactive oxygen species (ROS) among them potentially contribute to neurodegeneration. ${ }^{12}$ It is thus of obvious interest whether radical levels are significantly reduced in e-cigs and $\mathrm{HNB}$ products compared to conventional cigarettes. In particular for e-cigs, it has been recently pointed out that more research is required to understand, among other things, how designs and modes of use affect toxicity.

The technique of choice for radical detection in the particulate matter and gas phase derived from cigarette smoke is electron paramagnetic resonance (EPR) spectroscopy, $^{14}$ which is applied in combination with spin trapping ${ }^{15,16}$ for the short-lived radicals observed in the gas phase. Nitric oxide, which is also absent in the FDA list of HPHCs, is thought to be involved in radical formation in the gas phase and can be detected by EPR spectroscopy after trapping with $\mathrm{Fe}(\mathrm{II})$-bis (diethyldithiocarbamate) $\left(\mathrm{Fe}(\mathrm{II})-(\mathrm{DETC})_{2}\right){ }^{17}$ Although quantification by infrared spectroscopy may be preferable if only nitric oxide (NO) is concerned, we consider

Received: February 27, 2019

Published: April 1, 2019 
it as useful to study NO levels alongside those of other free radicals under the same conditions. Since smoke composition varies considerably with smoking regime, it is best practice to perform experiments with a smoking machine under standardized conditions. Recent research has been mostly performed according to the Health Canada (HC) Intense smoking regime, which is also recommended by the WHO. ${ }^{18}$ This regime has also been used in a recent study of analytes from the FDA list in the aerosol produced by HNB products ${ }^{8}$ and in comparison of the levels of such analytes between conventional cigarettes and e-cigs. ${ }^{19}$ Here we set out to provide such a comparison for free radicals and NO between the conventional research cigarette $3 \mathrm{R} 4 \mathrm{~F}$, two e-cigs with different aerosol heating methods, and an HNB product.

\section{MATERIALS AND METHODS}

2.1. Reagents and Materials. Analytical grade spin traps were purchased from Enzo Life Sciences, Inc. (Lausen, Switzerland): $N$ tert-butyl- $\alpha$-phenyl-nitrone (PBN), 5-tert-butoxy-carbonyl-5-methyl1-pyrroline- $N$-oxide (BMPO), sodium diethyldithiocarbamate trihydrate (DETC), 5,5-dimethyl-1-pyrroline- $\mathrm{N}$-oxide (DMPO), 5-diethoxyphosphoryl-5-methyl-1-pyrroline- $\mathrm{N}$-oxide (DEPMPO), 2,2-dimethyl-4-phenyl-2H-imidazole-1-oxide (DMPIO), 5-di(isopropoxyphosphoryl)-5-methyl-1-pyrroline- $\mathrm{N}$-oxide (DIPPMPO), and 2,2,4-trimethyl-2H-imidazole-1-oxide (TMIO). Spin trapping agents were stored at $-20{ }^{\circ} \mathrm{C}$ in their original packaging and after opening under inert atmosphere. All chemicals used in this work were of analytical grade.

The $44 \mathrm{~mm}$ Cambridge filter pads (purchased in bulk from Hollingsworth \& Vose Air Filtration Ltd., Waterford Bridge, Kentmere, LA8 9JJ, UK and cut to size by Alfaset, Rue des Terreaux 46-50, 2300, La Chaux-de-Fonds) were stored in an airtight plastic bag until use.

2.2. Smoking and Vaping Products. Tobacco and nontobacco products were provided by Philip Morris International R\&D (Neuchâtel, Switzerland). This included the 3R4F research cigarette (University of Kentucky, Lexington, Kentucky, USA), ${ }^{20}$ the tobacco heating system THS 2.2, marketed as IQOS, with complementary tobacco sticks HEETS (Amber label) and two e-cig products, namely Solaris XL (flavor: "Amber Bliss"; $19.3 \mathrm{mg} / \mathrm{mL}$ nicotine) and MESH (flavor: "Classic Tobacco High"; $19 \mathrm{mg} / \mathrm{mL}$ nicotine). Note that flavoring influences the free radical levels in the aerosol of e-cigs. ${ }^{21}$ The Solaris product uses a standard wick-and-coil heating, while the MESH product uses a controlled mesh heating. The 3R4F research cigarettes and HEETS tobacco sticks were long-term stored in the original packaging or in an airtight plastic bag. For both products, a stick/cigarette pack out of 10 was selected randomly, and all sticks/ cigarettes were used. When the pack was emptied, the next was randomly selected.

Three randomly selected e-cig cartomizers per product were stored in the original packaging under inert atmosphere until their first usage and then kept in a constant humidity chamber ( $58 \%$ relative humidity, $22{ }^{\circ} \mathrm{C}$ ). Solaris does not have an indicator for liquid level, and the consumers are told to "notice a reduction in flavor and vapor". Although generally the liquid in one cartomizer is sufficient for $2 \mathrm{~h}$ of continuous use, in order to avoid possible dry puffing effects, ${ }^{22}$ every cartomizer was used for only five experiments (equal to 60 puffs).

2.3. Preparation of Spin Trap Solutions. Fresh spin trap solutions were prepared on a daily basis for a set of experiments, consisting usually of four measurements (all four products). Solutions were kept at $-78{ }^{\circ} \mathrm{C}$ under inert atmosphere during the day and were thawed prior to use.

For measurements with the spin trap PBN, the analysis procedure of Goel et al. ${ }^{23}$ was followed, except for replacing tert-butylbenzene by toluene: approximately $273 \mathrm{mg}(1.54 \mathrm{mmol})$ of PBN was dissolved in $30.8 \mathrm{~mL}$ of deoxygenated toluene giving a concentration of $0.05 \mathrm{M}$. For BMPO, the content of one whole flask (approximately $50 \mathrm{mg}$, $0.25 \mathrm{mmol}$ ) was dissolved in $30.8 \mathrm{~mL}$ of deoxygenated toluene giving an approximate concentration of $8.14 \mathrm{mM}$. Spin trap solutions for preliminary experiments with other spin traps were prepared analogously for DMPO following the procedure used for PBN and for other traps following the procedure used for BMPO. Freshly prepared $\mathrm{PBN}$ solution featured a small background signal, which was found to be stable over a day. This contribution is negligible compared to other uncertainties discussed below.

A $5 \mathrm{mM}$ solution of $\mathrm{Fe}$ (II)-DETC in toluene was prepared according to a modified version of the procedure described by Santos et al. ${ }^{24}$ To the phosphate buffered aqueous solution $(0.1 \mathrm{M}, \mathrm{pH}=$ 7.4), $180.24 \mathrm{mg}$ ( $0.8 \mathrm{mmol}, 2$ equiv) of $\mathrm{Na}$-DETC $3 \mathrm{H}_{2} \mathrm{O}$ was added, and the solution was heated to $30{ }^{\circ} \mathrm{C}$. Under stirring, $55.60 \mathrm{mg}(0.2$ mmol, 1 equiv) of $\mathrm{FeSO}_{4} \cdot 7 \mathrm{H}_{2} \mathrm{O}$ was slowly added. The resulting brown precipitate of $\mathrm{Fe}-(\mathrm{DETC})_{2}(70.48 \mathrm{mg}, 0.2 \mathrm{mmol})$ was extracted several times (typically $3 \times 7 \mathrm{~mL}$ followed by $4 \mathrm{~mL}$ and then $3 \mathrm{~mL}$ ) with toluene giving a clear aqueous phase and an orange residue. The combined toluene fractions of dark brown/black color were dried under reduced pressure and stored overnight at $+4{ }^{\circ} \mathrm{C}$. Prior to usage, the residual solid was solved in $40 \mathrm{~mL}$ of toluene giving the desired solution of $\mathrm{Fe}(\mathrm{II})-(\mathrm{DETC})_{2}$.

2.4. Generation of Smoke and Aerosols. The products were smoked using a single-port smoking machine. This smoking machine and the aerosol trapping device consisted of an electric contact-free cigarette lighter (1), a fishtail chimney for capturing sidestream smoke based on the Health Canada normative T-212 (2), a cigarette holder and particulate phase trap (to hold a Cambridge filter pad to conform with the ISO 3308 norm (3), two consecutive impingers containing the spin trapping solutions (4), and a programmable single syringe pump manufactured by Burghart Messtechnik GmbH, Germany (5). Specifically, the electronic lighter is composed of a halogen lamp and a quartz rod which can be automatically moved to be positioned in front of the cigarette. When the lamp is lit, the quartz rod directs the infrared light to the cigarette end heating it up until it starts burning. In preliminary tests, a distance of approximately $3 \mathrm{~mm}$ between the quartz rod and the cigarette end was found to be good to light the cigarette. Deviation of the cigarette position from the central axis of the rod is detrimental to homogeneous lighting of the cigarette. The first puff is drawn when smoke is visible and the lighter is deactivated directly after the first draw. The advantage of the electric lighter is repeatability and that the sidestream smoke can be captured immediately after ignition. The particulate phase trap can be removed to perform measurements of the whole aerosol. The pump can be programmed to perform according to different smoking regimes, however, only full puffs can be taken, that is, a puff cannot be stopped prematurely as specified by the ISO 4387 norm. A photograph and schematic drawing of the setup are shown in Figure S1.

Prior to smoking, 3R4F cigarettes were conditioned in a humidity chamber at a relative humidity of $58 \%$ and a temperature of approximately $22{ }^{\circ} \mathrm{C}$ for at least $48 \mathrm{~h}$ and a maximum of 10 days, according to the ISO 4387 norm. Cigarettes were smoked according to the HC Intense regime T-115 up to the standard butt length of 33 $\mathrm{mm}$. This involves a puff volume of $55 \mathrm{~mL}$ over a duration of $2 \mathrm{~s}$ with a sinusoidal puffing profile. Two puffs were taken every minute with two additional immediate clearing puffs (no waiting time between them) taken after the cigarette is disposed of. Two clearing puffs are sufficient to draw several times the volume between the cigarette holder and the first impinger. Sidestream smoke was captured by the fishtail chimney surrounding the burning cigarette, a Cambridge filter pad on top of the chimney, and an additional Soxhlet filter. The air flow rate through the fishtail chimney is set up to be $3 \mathrm{~L} / \mathrm{min}$ according to HC T-212.

The aerosol of HEETS sticks and e-cigs was generated according to Cooperation Centre for Scientific Research Relative to Tobacco (CORESTA) recommendation no. 81: A rectangular puffing profile was used to draw a volume of $55 \mathrm{~mL}$ over a duration of $3 \mathrm{~s}$ at a frequency of two draws every minute. Twelve puffs were drawn in total. The first puff was taken when the product was ready (i.e., preheated) according to individual operations manuals. No sidestream smoke capturing was performed since no smoldering in between puffs occurs. All products were preconditioned in the same fashion as the 


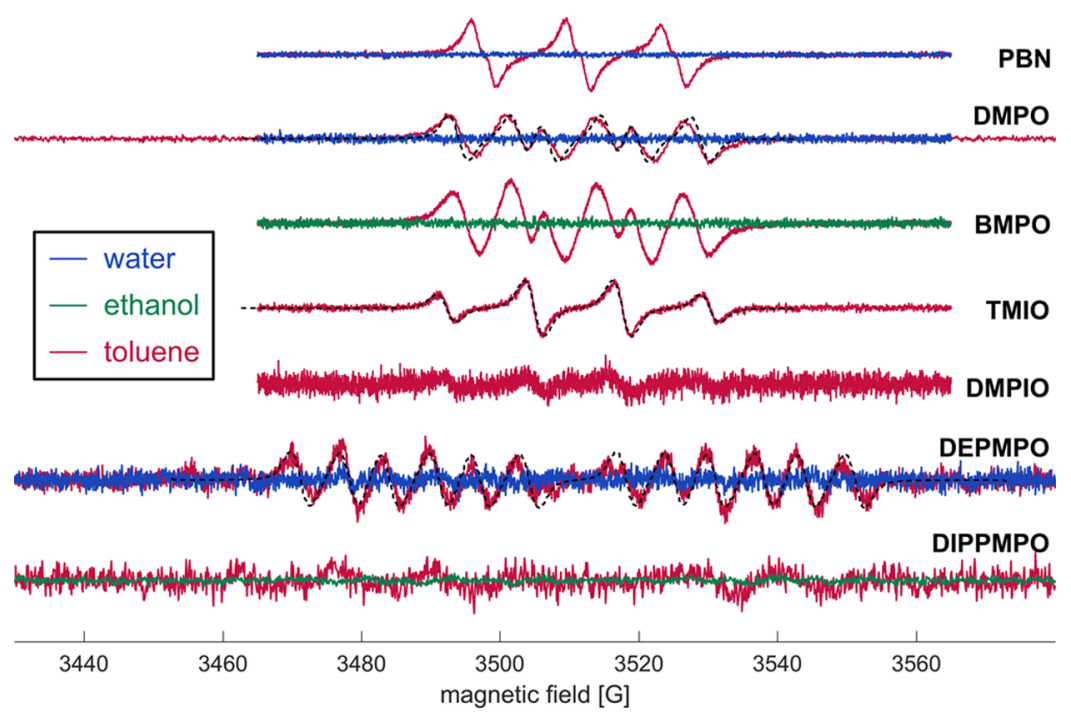

Figure 1. Spectra of gas-phase radicals of 3R4F smoke trapped by different spin traps. Solvents are indicated by color: water $=$ blue, toluene $=$ red, and ethanol $=$ green. Fits or simulations are shown as black dashed lines. From top to bottom (concentration of spin traps in brackets): PBN in water $(20 \mathrm{mM})$ and toluene $(20 \mathrm{mM})$; DMPO in water $(20 \mathrm{mM})$ and toluene $(40 \mathrm{mM})$, simulation with values for $\bullet \mathrm{OC}^{2}\left(\mathrm{CH}_{3}\right)_{2} \mathrm{C}_{6} \mathrm{H}_{5} ;{ }^{27} \mathrm{BMPO}$ in ethanol $(7.3 \mathrm{mM})$ and toluene $(10 \mathrm{mM})$; TMIO in toluene $(5.7 \mathrm{mM})$ and fit; DMPIO in toluene $(10 \mathrm{mM})$; DEPMPO in water (2.2 $\mathrm{mM})$ and toluene $(0.87 \mathrm{mM}$, recorded $30 \mathrm{~min}$ after smoking, and fit); and DIPPMPO in ethanol $(6.3 \mathrm{mM})$ and toluene $(8 \mathrm{mM})$.

3R4F cigarettes with the only difference that e-cig cartomizers were used directly after opening from the sealed packaging and were kept afterward in the conditioning box for further experiments. Due to the flat shape of the MESH-mouth piece, a home-built adapter was used for inserting this e-cig into the cigarette holder. The adapter was built from a short glass tube which was connected to the mouth piece with a wide silicon hose.

Blanks were measured by drawing air through the impingers either directly (air blank, measured twice) or through the cigarette holder and Cambridge filter (machine background, measured once). Differences between the background signals were within their uncertainty. Hence, we combined all three background measurements for computing a mean background and its uncertainty.

All products were used immediately after removing from the conditioning chamber. After the last puff, the products were kept in place for a further $30 \mathrm{~s}$ for residual smoke/aerosol deposition. In order to minimize contaminations between products, each product used its own set of labyrinth seals which are found in the cigarette holder.

2.5. Puff Volume Determination. Puff volume was measured according to ISO 4387:2000 with a soap bubble flow meter. A bubble flow meter was designed using a graduated measuring glass (precision $\pm 0.5 \mathrm{~mL}$ ) attached to a silicon hose (Figure S2). A soap solution was made from liquid hand soap (approximately $20 \mathrm{~mL}$ ), water (approximately $40 \mathrm{~mL}$ ), and a sugar cube for stabilizing bubbles. The real puff volume was determined for the apparatus setup for gasphase analysis, whole smoke analysis, and for gas-phase analysis with only one impinger and then adjusted by reprogramming the pump to give puff volumes of $55-55.5 \mathrm{~mL}$.

2.6. EPR Measurements. Unless noted otherwise, the spectra were taken using an Bruker EleXsys E500 X-band spectrometer with the following parameters: microwave frequency, $\sim 9.88 \mathrm{GHz}$; modulation frequency, $100 \mathrm{kHz}$; microwave power, $2.01 \mathrm{~mW}(20$ $\mathrm{dB}$ attenuation); scan range, $100 \mathrm{G}$; modulation amplitude, $2 \mathrm{G}$; sweep time, $10.49 \mathrm{~s}$; time constant, $1.28 \mathrm{~ms}$; conversion time, 5.12 ms; and receiver gain, $50 \mathrm{~dB}, 2048$ points. In order to ensure highly reproducible spin count measurements, we used an AquaX capillary bundle setup (four capillaries, Bruker Biospin $\mathrm{GmbH}$, Karlsruhe, Germany). Variations in sample volume and position, which were found to be significant when changing sample tubes, ${ }^{25}$ can thus be avoided, albeit at the expense of somewhat lower total sample volume and correspondingly lower sensitivity. The EPR measurement setup is shown in Figure S3. AquaX is vertically installed inside the resonator (2; red dashed line). New sample solutions were injected with a 10
$\mathrm{mL}$ syringe from the bottom, and the syringe was kept in place as a sealing device (1). The teflon tubing coming from the top directed the samples into a waste container (3).

Prior to each smoking or vaping experiment, a background measurement of the spin trap solution was taken by injecting $3 \mathrm{~mL}$ of the spin trap solution into the AquaX. Measurements were performed at room temperature $\left(21^{\circ} \mathrm{C}\right)$. Preliminary experiments had shown that with this volume, the contribution of residue from previous experiments and dilution due to nonlaminar flow during sample exchange became negligible. Spin concentrations were determined by double integration and comparison with a calibration spectrum of 4-hydroxy-2,2,6,6-tetramethylpiperidin-1-oxyl (TEMPOL) solutions. For that, solutions with TEMPOL concentrations of $0.05,0.5,5$, and $50 \mu \mathrm{M}$ were prepared and measured. A linear fit of the logarithm of the double integral versus the logarithm of the concentration had an excellent $R^{2}$ value of 0.9999 . Spectra were simulated or fitted using the MATLAB toolbox EasySpin. ${ }^{26}$ Assignments based on the hyperfine couplings were made using the $\mathrm{NIH}$ spin trap database.

2.7. Preparation of Aerosol Radical Solutions. Mainstream smoke or aerosol was passed into two sequential impingers $(25 \mathrm{~mL}$ volume), each filled with $4 \mathrm{~mL}$ of the spin trap solutions. When the second impinger was not analyzed, the spin trap solution was replaced by pure toluene. The connections between the smoke machine and the first impinger as well as between the first and the second impinger were made of glassware and kept as short as possible in order to trap radicals as fast as possible. In total, the distance between cigarette butt end (or product mouth piece) and the surface of the solution in the first impinger was approximately $32.5 \mathrm{~cm}$. Inside the tubing, the gas flow rates were $r_{\mathrm{HC}}=218 \mathrm{~cm} / \mathrm{s}$ for the $\mathrm{HC}$ intense smoking regime and $r_{\mathrm{COR}}=146 \mathrm{~cm} / \mathrm{s}$ for the CORESTA regime. Based on these flow rates, radicals were expected to reach the surface of the spin trap solution after $t_{\mathrm{HC}}=0.149 \mathrm{~s}$ and $t_{\mathrm{COR}}=0.223 \mathrm{~s}$, respectively.

If the passing aerosol led to a reduction of toluene volume, then this volume was adjusted. For immediate measurements without additional deoxygenation, $3 \mathrm{~mL}$ of the spin trap solution was taken out and injected into the AquaX. The time between the last puff and the start of the EPR measurement was $5 \mathrm{~min}$.

For measurements on deoxygenated solutions (vide infra), approximately $4 \mathrm{~mL}$ of the solution was transferred into a Schlenk flask, and three freeze-pump-thaw cycles were performed. Briefly, the solution was frozen using liquid nitrogen, and a vacuum was subsequently applied. The solution was then thawed so that dissolved 
gases could leave the liquid. Afterward, gaseous nitrogen was introduced into the flask, and the procedure was repeated two more times. Then, $3 \mathrm{~mL}$ of the deoxygenated solution was injected into the AquaX. With this procedure, the time between the last puff and the start of the first EPR measurement was $20 \mathrm{~min}$.

2.8. Weight of Total Particulate Matter and Preparation of Particulate Phase Radical Solutions. Particulate phase of the mainstream aerosol was trapped by a conditioned (58\% relative humidity, $22{ }^{\circ} \mathrm{C}$, at least $12 \mathrm{~h}$ in the conditioning box according to CORESTA method no. 23) Cambridge filter pad which was inserted into the particulate phase trap. Immediately after smoking or vaping, the smoke trap was removed and sealed from both sides, as recommended by CORESTA method no. 23 and HC method T-212. The filter was weighed together with the seals and the smoke trap, and the total particulate matter was determined by subtracting the weight of the apparatus prior to smoking.

The filter was then removed from the smoke trap with tweezers and folded two times, with the collection side being on the inside. The two clean outer quarters were used to collect any residual particulates in the smoke trap (front side and back side). The filter was then extracted with $10 \mathrm{~mL}$ of pure toluene for $20 \mathrm{~min}$ by gently shaking the Erlenmeyer flask. Afterward, approximately $4 \mathrm{~mL}$ of the extract was transferred into a Schlenk flask, and three cycles of freeze-pumpthaw were performed. Then, $3 \mathrm{~mL}$ of the deoxygenated solution was inserted into the AquaX.

\section{RESULTS}

3.1. Choice of Spin Traps, Solvent, and Spin Trap Concentration. Efficiency of spin trapping and stability of the radical adducts in solution depend on the combination of spin trap and radical as well as on solvent. ${ }^{16}$ Therefore, we have tested seven spin probes and three solvents (Figure 1). The best sensitivity was obtained with PBN and BMPO, which were therefore selected for detailed studies.

For the spin traps that we did not use in further studies, we simulated or fitted the spectra obtained. For DMPO, the spectrum is rather well reproduced by a simulation with the hyperfine parameters $A_{\mathrm{N}}=13.08 \mathrm{G}$ and $A_{\mathrm{H}}=8.88 \mathrm{G}$ reported for $\bullet \mathrm{OC}\left(\mathrm{CH}_{3}\right)_{2} \mathrm{C}_{6} \mathrm{H}_{5},{ }^{27}$ leading us to a tentative assignment to alkoxy radicals, although organic peroxy radicals cannot be fully excluded with this spin trap. The spectrum for TMIO is fitted perfectly with $A_{\mathrm{N}}=12.7 \mathrm{G}$ and $A_{\mathrm{H}}=12.7 \mathrm{G}$. This excludes carbon-centered radicals and again suggests an assignment to oxygen-centered radicals. A more definite assignment cannot be made for TMIO because of a lack of published data for comparison, especially for trapping in nonpolar solvents. A reasonable fit of the spectrum obtained with DEPMPO is obtained with $A_{\mathrm{P}}=47 \mathrm{G}, A_{\mathrm{N}}=13 \mathrm{G}$, and $A_{\mathrm{H}}$ $=7 \mathrm{G}$, which is close to the values for the major conformers of DEPMPO-trapped $\bullet$ OMe, $\bullet \mathrm{OEt}$, and $\bullet \mathrm{OBu}$ radicals in hexane. ${ }^{28}$ The same study shows that the $A_{\mathrm{N}} / A_{\mathrm{H}}$ ratio differs significantly between alkoxy and alkylperoxy radicals for DEPMPO. All of the results for the spin traps discussed so far are thus consistent with the main trapped radical species being alkoxy radicals under the conditions used by us. Assignments for the BMPO and PBN spins traps are discussed below on the basis of fitting better-resolved spectra obtained in deoxygenated solution. Considerations on the deoxygenation method are described in the Supporting Information.

3.2. Trapping Efficiency and Radical Adduct Stability. The amount of spin trap that we used was by far sufficient to trap all free radicals in smoke according to the amount of radicals reported in previous studies. However, the procedure involved two steps that could cause incomplete trapping. First, radicals that are originally in the gas phase must enter the liquid phase in the impinger, and, second, the dissolved radicals must react much faster with the spin trap than they decay in solution. In order to test for the completeness of trapping, we have used two impingers with spin trap solution and measured the solution from both impingers with a time delay of $30 \mathrm{~min}$. In order to estimate the effects of spin adduct decay during the lag time, the experiment was performed twice, measuring a BMPO solution after trapping of gas phase radicals from 3R4F cigarette smoke from the first impinger first and after measuring the solution from the second impinger first (Figure S5).

In both cases, the solution in the first impinger was found to contain more radicals, but the intensity ratio was larger when this solution was measured first, indicating a significant adduct decay within $30 \mathrm{~min}$. The double integral of the EPR spectrum of the solution in the second impinger was $23 \%$ of the one in the first impinger if the first impinger was measured first, but $33 \%$ if the second impinger was measured first. Closer inspection revealed that the spectral line shape changes during decay, indicating that at least two adducts with different stability were observed. Precise quantification of the trapping efficiency would thus be involved, and it would require an estimate of the loss of radicals in the gas phase between impingers 1 and 2 . However, if we neglect this loss and specify an average trapping efficiency for all different radicals, we arrive at an estimate of $72 \%$. This compares to values reported for PBN in benzene of $47 \%{ }^{29}$ and $95 \%,{ }^{25}$ where the former study had used larger spin trap concentrations and volumes than the latter one. We can conclude that uncertainty in the trapping efficiency and the decay of the various radical adducts were the main source of error in estimating the total number of free radicals in cigarette smoke.

3.3. Whole Smoke or Gas Phase? Since the whole smoke is inhaled, it would appear better to characterize free radicals in whole smoke rather than in the gas phase separated by a Cambridge filter. However, early experiments by Pryor et al. had failed to trap radicals from whole smoke with PBN in benzene solution. ${ }^{30}$ Our repetition of this experiment with PBN in toluene confirmed this result (Figure S6). In contrast, quantification of $\mathrm{NO}$ with the $\mathrm{Fe}(\mathrm{II})-(\mathrm{DETC})_{2}$ complex was possible in both whole smoke and the separated gas phase. From a physiological point of view, radicals observed after removing particulate matter and absorbing the gas phase in an nonpolar solvent should be considered as potentially relevant. The amount of particulate matter decreases during inhalation through deposition as the aerosol travels down the respiratory tract to the alveoli. Furthermore, the hydrophobic tails of pulmonary surfactant molecules that are in contact with the air in alveoli mimic an nonpolar solvent. The Cambridge filters used for separating particulate matter are unlikely to contribute to free radical generation.

3.4. Further Considerations on Reliable Comparison of Radical Levels. In order to exclude as many confounding factors as possible, the same experiment was performed on all four products on the same day with the same spin trap solution. Previous work on e-cigs ${ }^{31}$ and on an early HNB product $^{32}$ indicate much lower levels of radicals than in conventional cigarettes. To avoid contamination from an earlier measurement, we performed the series of experiments always in the order of expected increasing radical level, starting with the two e-cigs in random order, continuing with IQOS, and finishing with the 3R4F research cigarette. All spin trapping experiments were performed in triplicate. For 

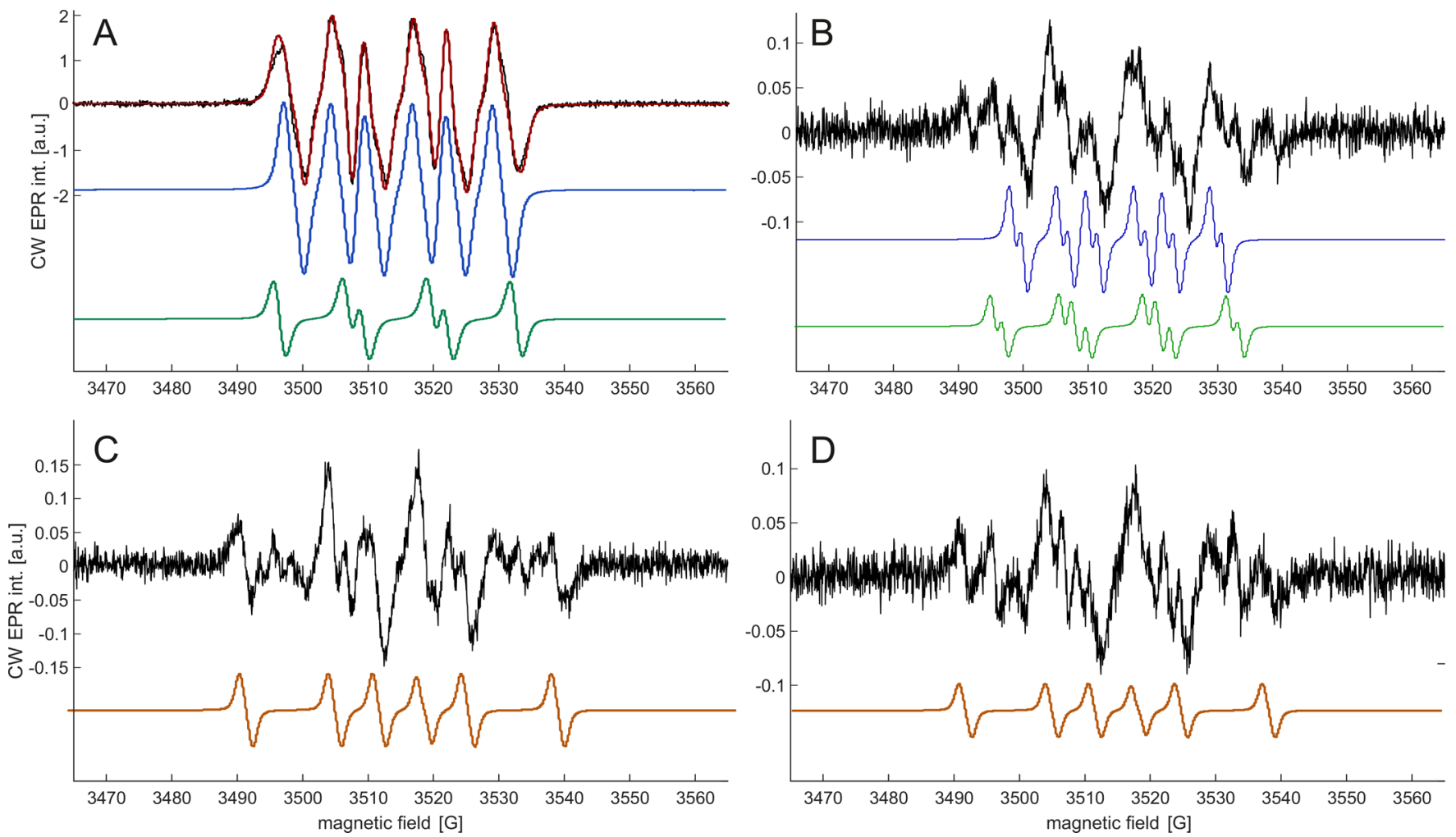

Figure 2. EPR spectra of solutions of $8.14 \mathrm{mM}$ BMPO in toluene from the first impinger after passing the gas phase of the aerosol from different products through the spin-trap solution. Experimental spectra are shown in black, fits in red, and component spectra in blue, green, and orange. (A) 3R4F research cigarette and two-component fit; (B) IQOS (HNB product) and two of three component spectra (see text); (C) MESH (e-cig) and one of three component spectra (see text); and (D) Solaris (e-cig) and one of three component spectra (see text).
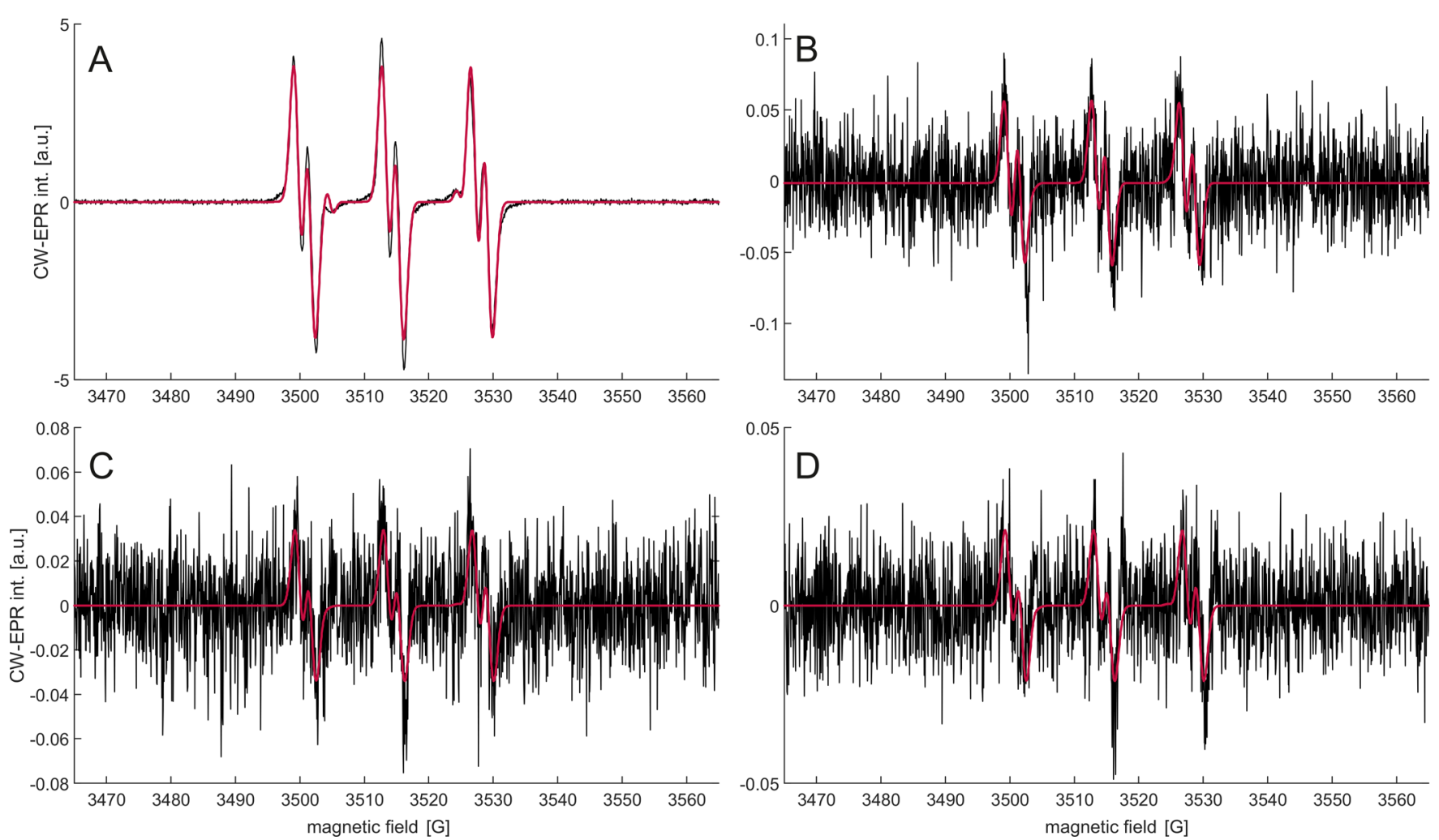

Figure 3. EPR spectra of solutions of $50 \mathrm{mM} \mathrm{PBN}$ in toluene from the first impinger after passing the gas phase of the aerosol from different products through the spin-trap solution. Experimental spectra are shown in black and best fits by EasySpin in red. (A) 3R4F research cigarette; (B) IQOS (HNB product); (C) MESH (e-cig); and (D) Solaris (e-cig). 
measurements where the radical level was significantly above the air background, mean values and standard deviation are reported. Only the mean value is reported if the spin count is indistinguishable from the air background.

3.5. Spin Trapping with BMPO. BMPO was used for spin trapping, since initial experiments indicated a larger concentration of radicals trapped from 3R4F smoke with BMPO compared to PBN. The spectrum obtained with 3R4F smoke can be reasonably well fitted (Figure $2 \mathrm{~A}$ ) with two components with hyperfine parameters of $A_{\mathrm{N}}=12.5 \mathrm{G}, A_{\mathrm{H} 1}=7.4 \mathrm{G}$, and $A_{\mathrm{H} 2}=1.6 \mathrm{G}(78.4 \%$ of the double integral $)$ and $A_{\mathrm{N}}=12.9 \mathrm{G}$ and $A_{\mathrm{H}}=10.6 \mathrm{G}(21.6 \%)$. Both radicals are certainly oxygencentered. By analogy with the case of DMPO, they could be assigned to alkoxy and peroxy radicals, ${ }^{33}$ although it cannot be excluded that they are two conformers of an adduct originating from only one of these species.

Spectra of the vaping products were more complex and exhibited contributions of at least three species. We refrained from line shape fitting, since the obtained parameters sets with three species were not unique at the signal-to-noise ratio that we could achieve. We can remark, however, that all features could be assigned to the same two species observed with $3 \mathrm{R} 4 \mathrm{~F}$ smoke, which we simulated with lower line width in Figure 2B and to a third species with $A_{\mathrm{N}}=13.4 \mathrm{G}$ and $A_{\mathrm{H}}=20.0 \mathrm{G}$ and slightly lower $g$ value (simulation in orange color in Figure $2 \mathrm{C}, \mathrm{D})$ that can be assigned to a carbon-centered radical. We refrained from using BMPO for quantification, as the amount of trapped radicals was poorly reproducible with this trap (see Table S1).

3.6. Spin Trapping with PBN. With PBN, a spin adduct signal was detected in all four products, albeit with strongly different intensities (Figure 3). Unlike for BMPO, quantification is satisfyingly reproducible upon repetition of the experiment. Because of the very weak spectra, we compared to the air background measurements (Table 1). We find that

Table 1. Measurements of Gas-Phase Radical Levels in Different Smoking and Vaping Products with the PBN Spin Trap in Toluene ${ }^{a}$

\begin{tabular}{lll} 
aerosol & rel. int. & \multicolumn{1}{c}{$n[\mathrm{nmol}]$} \\
3R4F & 1 & $25.9 \pm 0.6$ \\
IQOS & 0.016 & $0.394 \pm 0.055$ \\
MESH & 0.010 & 0.251 \\
Solaris & 0.008 & 0.193 \\
air & 0.010 & 0.256
\end{tabular}

${ }^{a}$ Shown are the relative double integral of the signal with respect to the signal of 3R4F research cigarettes and the amount of radicals $n$ trapped per cigarette, e-cig, or IQOS tobacco stick.

the signals from the e-cigs are not significantly different from the air background at our measurement conditions and that the signals from IQOS exceed the air background by only a factor of about 2 .

The signals can be simulated assuming two spin adducts, one having hyperfine couplings typical for oxygen-centered radicals $\left(A_{\mathrm{N}}=13.8-13.9 \mathrm{G}, A_{\mathrm{H}}=1.8-1.9 \mathrm{G}\right)$ and the other, much weaker one being the oxidation product of $\mathrm{PBN}$, the acyl nitroxide PBNOx $\left(A_{\mathrm{N}}=10.0-10.6 \mathrm{G}\right)$. The former radical adduct has parameters in agreement with the ones reported for PBN-trapped alkoxy radicals in toluene $\left(A_{\mathrm{N}}=13.7 \mathrm{G}, a_{\mathrm{H}}=1.8\right.$ $\mathrm{G}){ }^{34}$ although a different identity of the radical cannot be completely excluded. The assignment to alkoxy radicals with
DEPMPO, mentioned above, strongly suggests that PBN also traps alkoxy radicals. Note that the acyl nitroxide can be formed by reaction of PBN with $\mathrm{NO}_{2}{ }^{35}$ which is an expected component in cigarette smoke (vide infra).

For the 3R4F case, spin adduct decay kinetics was fitted by an exponential decay of the form $[\mathrm{R}](t)=[\mathrm{R}](0) \times \mathrm{e}^{-k t}$, where $t$ is the time and $k$ the decay rate constant. Amplitude of the main signal was used as the quantity proportional to radical concentration $[\mathrm{R}]$ in order to avoid contamination of the double integral by the PBNOx signal, which increases with time. We found $k=(2.09 \pm 0.23) \times 10^{-4} \mathrm{~s}^{-1}$, corresponding to a half-life time $t_{1 / 2}=(3350 \pm 357) \mathrm{s}$. This relatively high stability is consistent with an assignment to alkoxy radicals. Signals of the HNB product and of the e-cigs were too weak to analyze kinetics. The signals are near the detection limit and consistent with the first component that we assigned to alkoxy radicals in the $3 \mathrm{R} 4 \mathrm{~F}$ case.

3.7. Trapping of $\mathrm{NO}$ with $\mathrm{Fe}(\mathrm{II})-(\mathrm{DETC})_{2}$. The $\mathrm{NO}$ trapping experiments were performed with $\mathrm{Fe}(\mathrm{II})-(\mathrm{DETC})_{2}$ in toluene in order to connect them to the spin trapping experiments performed in the same solvent. They yielded detectable signals only in measurements of 3R4F cigarettes and of the HNB product IQOS (Figure 4). The expected broad

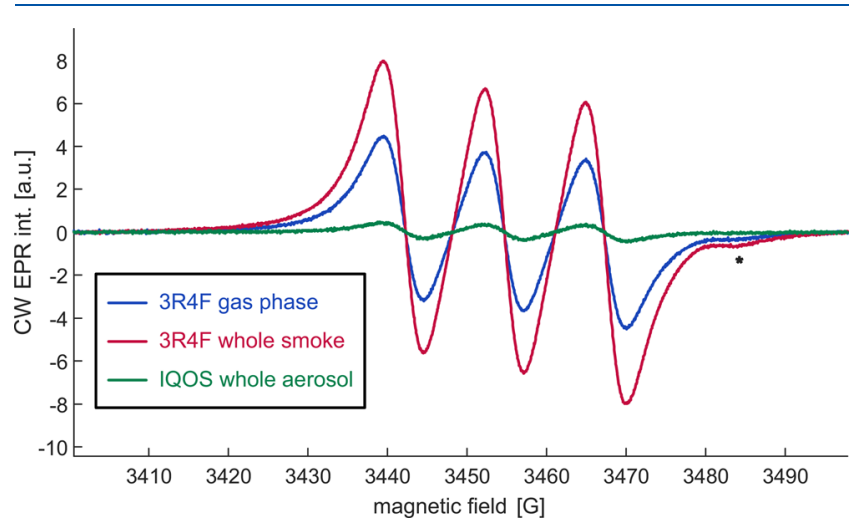

Figure 4. Typical spectra obtained by NO trapping with Fe(II)(DETC $)_{2}$ of whole smoke of the $3 \mathrm{R} 4 \mathrm{~F}$ research cigarette and whole aerosol of IQOS. A spectrum of NO captured from the gas phase of 3R4F smoke is shown for comparison. The identity of the signal marked with an asterisk is unknown.

triplet with a nitrogen hyperfine coupling $a_{\mathrm{N}}=(12.75 \pm 0.08)$ $\mathrm{G}$ was observed, which compares to coupling of $12.5 \mathrm{G}$ in DMSO $^{16}$ and $12.7 \mathrm{G}$ in water. ${ }^{36}$ An additional signal was observed at the high-field edge of the spectrum (asterisk). Given the width and much higher amplitude of the main spectrum, this signal could not be further analyzed.

Relative intensities and NO masses are reported in Table 2. For NO, whole smoke measurements on 3R4F research cigarettes detect a larger concentration than gas-phase measurements (reduction to $75 \%$ ). The amount of trapped NO increases with time from about $75 \%$ of the whole-smoke signal at the start of the measurement to about $168 \%$ of this signal in the long-time limit. The kinetics of this increase is discussed in the Supporting Information. The NO concentration in IQOS whole aerosol is approximately $7.3 \%$ of the level observed in whole cigarette smoke.

3.8. Total Particulate Matter. The total particulate matter (TPM) was weighed, and EPR spectra of toluene extracts were acquired. By comparing these spectra with background spectra 
Table 2. Measurements of NO Mass in the Aerosol of 3R4F Research Cigarettes and the HNB Product IQOS (whole aerosol, WA) ${ }^{a}$

$\begin{array}{lll}\text { experiment } & \text { rel. int. } & m[\mu \mathrm{g}] \\ \text { 3R4F (WS) } & 1 & 16.4 \pm 4.7 \\ \text { 3R4F (GP,1) } & 0.78 & 12.8 \\ \text { 3R4F (GP,2) } & 0.73 & 11.9 \\ \text { IQOS (WA) } & 0.073 & 1.17 \pm 0.14\end{array}$

${ }^{a}$ No detectable signal was found for e-cigs. Shown are the relative double integral of the signal with respect to the signal of whole smoke (WS) of 3R4F research cigarettes and the mass of NO. Since only two gas-phase (GP) measurements were performed, they are reported individually instead as an average.

obtained from an extract of clean Cambridge filter pads, we found that signals were generally weak and even signals obtained from the aerosol from three cigarettes were not consistently above background for IQOS and Solaris. For 3R4F, we measured $(0.95 \pm 0.25)$ nmol radicals per cigarette. For IQOS, Solaris, and MESH, we can only specify an upper limit of $0.07 \mathrm{nmol} /$ cigarette that roughly corresponds to our detection limit for the broad signal with a total line width of 30 G (Figure S7).

The weight of TPM per cigarette was similar for all products. We find $(32.6 \pm 6.1) \mathrm{mg}$ TPM for 3R4F cigarettes, $(26.1 \pm$ $1.3) \mathrm{mg}$ for IQOS, $(23.8 \pm 1.2) \mathrm{mg}$ for Solaris, and $52.4 \mathrm{mg}$ for $\mathrm{MESH}$. Since the experiment was done only twice for MESH, we do not report an uncertainty, but the difference of the two experiments (56.7 and $48.1 \mathrm{mg}$ ) indicates a similar experimental error as in the other cases.

\section{DISCUSSION}

4.1. Radical Levels in the Gas Phase. Relative radical levels can be quantified from the measurements made using PBN. With $(25.9 \pm 0.6) \mathrm{nmol} /$ cigarette, we find a three times higher radical level for 3R4F research cigarettes than Goel et al., who found $(8 \pm 2) \mathrm{nmol} /$ cigarette by following the ISO norm $^{23}$ instead of the HC intense smoking regime. Indeed, for a filter ventilation of $38 \%$, as found for $3 \mathrm{R} 4 \mathrm{~F}$ by Goel et al. or of $29 \%$ as reported by Roemer et al., ${ }^{20}$ Counts et al. ${ }^{37}$ have predicted 2-4 times higher emissions from the $\mathrm{HC}$ intense regime than from the ISO regime. Based on the time passed between the end of smoking and the decay rate determined in kinetics experiments, we can estimate an initial radical amount of $33.3 \mathrm{nmol} /$ cigarette. This amount is still much lower than the $225 \mathrm{nmol}$ estimated from whole smoke solid-phase trapping $^{38}$ or the $70 \mathrm{nmol}$ estimated from whole-smoke trapping in toluene by nitroxides. ${ }^{39}$ In both of these wholesmoke studies, adducts were quantified by fluorescence detection after a separation by HPLC.

For both e-cigs, radical levels are of the order of air background, and for the HNB product IQOS, they are near the upper limit of such background. Furthermore, the air blank EPR spectra (Figure S8) are very similar to the spectra measured with e-cig or IQOS aerosol. We cannot exclude that the background signal is induced by environmentally persistent free radicals in airborne particulate matter. ${ }^{40}$ This underscores the importance of background measurements that was pointed out by Margham et al. ${ }^{31}$ and suggests that the radical levels that we found for e-cigs and the HNB product must be understood as upper limit levels. Results from quantitative spin trapping with PBN indicate that the free radical level in aerosol from the
HNB product IQOS does not exceed $1 \%$ of the level observed for 3R4F research cigarettes, which is in agreement with a study by Pryor et al. on a very early HNB product. ${ }^{32}$ This reduction compared to $3 \mathrm{R} 4 \mathrm{~F}$ research cigarettes is on a similar level as the $1-3 \%$ found with THS 2.2 for volatile organic compounds. ${ }^{8}$

4.2. NO Levels in Whole Smoke and in the Gas Phase. No EPR signal due to trapped NO could be detected for e-cigs. This is consistent with findings by Margham et al. on the Vype ePen e-cig ${ }^{31}$ and not surprising, since the sources of NO in cigarette smoke are nitrates and tobacco proteins, which are missing in e-cigs. These NO sources do exist in HNB products, and indeed we found a significant NO level for IQOS, which is in qualitative agreement with earlier results, which found an abundance of $2-3 \%$ compared to the $3 R 4 F$ cigarette. ${ }^{41,42}$ In contrast, the NO abundance compared to $3 \mathrm{R} 4 \mathrm{~F}$ cigarette is $7.3 \%$ in our study if we consider the first EPR measurement and $5.5 \%$ if we consider the saturation level of trapped NO. In both earlier studies, IQOS was operated using the $\mathrm{HC}$ intense regime, providing a very direct comparison with the $3 \mathrm{R} 4 \mathrm{~F}$ cigarette. In contrast, we operated IQOS according to the CORESTA recommendation for electronic devices. Both regimes use the same puff volume and frequency and differ only in puff duration and profile. Furthermore, both earlier studies measured $\mathrm{NO}$ in the gas phase that had passed a Cambridge filter pad, whereas we measured whole smoke and aerosol.

As seen in Figure S9, NO saturation values for the 3R4F cigarette are higher for our gas-phase measurement than for our whole smoke measurements, while initial values are lower. A possible explanation is an influence of particulate matter on the reaction between $\mathrm{NO}, \mathrm{O}_{2}$, and $\mathrm{NO}_{2}$ in the gas phase, $\mathrm{NO}_{2}$ absorption by the trap solution, and slow reduction of $\mathrm{NO}_{2}$ to NO in this solution.

EPR spectroscopy of IQOS aerosol after trapping in a toluene solution of $\mathrm{Fe}(\mathrm{II})$-(DETC) $)_{2}$ provided an absolute value of $1.17 \mu \mathrm{g} /$ tobacco stick that is an order of magnitude lower than previously reported values of $13.0 \mu \mathrm{g}$ measured with an $\mathrm{NO}$ meter ${ }^{42}$ and of $16.8 \mu \mathrm{g}$ measured according to a $\mathrm{HC}$ official method for NO determination. ${ }^{41}$ Likewise, measurements on the 3R4F research cigarette by other techniques provided much higher NO levels of $510 \mu \mathrm{g}$, $(491 \pm 12) \mu \mathrm{g},{ }^{42}$ and $(503 \pm 23) \mu \mathrm{g} .{ }^{31}$ Although NO is an nonpolar gas, we cannot be sure that it is fully absorbed by the solution in the impinger. The other techniques collect the aerosol in a gas bag and are thus not affected by a corresponding problem. Therefore, we consider the absolute NO amounts measured by EPR spectroscopy as less reliable than those measured by other techniques. This assessment is supported by the NO amount of $7.57 \mu \mathrm{g}$ measured for a Japanese cigarette by Shinagawa et al. with a different NO trap in aqueous solution, ${ }^{36}$ which is even lower than our value for the $3 \mathrm{R} 4 \mathrm{~F}$ cigarette. In that case, the lower yield that they obtained compared to ours may be explained by the use of a polar solvent, but it cannot be excluded either that it resulted from a nonstandard smoking procedure using a continuous air flow of $600 \mathrm{~mL} / \mathrm{min}$.

4.3. Analysis of the Particulate Phase. The TPM weight for the 3R4F research cigarette of $(32.6 \pm 6.1) \mathrm{mg}$ agrees quite well with the $(37.7 \pm 0.3) \mathrm{mg}$ reported by Roemer et al., ${ }^{17}$ but is significantly lower than the values of $(49 \pm 1.5) \mathrm{mg}^{42}$ and $44.7 \mathrm{mg}^{41}$ reported by others. It is unclear why these values differ that much, as in all cases, the $\mathrm{HC}$ intense regime was 
used. We found that smoking two or three cigarettes on the same Cambridge filter pad already reduced the TPM yield, except for the Solaris e-cig. A comparison of our IQOS data of $(26.1 \pm 1.3) \mathrm{mg}$ to the $(48.2 \pm 0.8) \mathrm{mg}^{42}$ and $(54.7 \pm 3.2)$ $\mathrm{mg}^{41}$ found by others shows the same trend. However, it can be concluded that all products yield similar amounts of TPM. In our hands, the MESH e-cig produced more TPM than the other e-cig product Solaris, which could be attributed to the different heating method.

We find long-lived radicals at a level of $(0.95 \pm 0.25) \mathrm{nmol}$ in the TPM extract of 3R4F cigarettes, which is more than an order of magnitude higher than the value of $(64 \pm 13)$ pmol reported by Goel et al. ${ }^{23}$ We have no explanation for this discrepancy, except that Goel et al. used the ISO regime, whereas we used the HC intense regime.

In contrast, free radicals in TPM from a single e-cig or IQOS tobacco stick are below the level that allows for a reliable quantification by EPR spectroscopy $(0.7 \mathrm{nmol})$. This agrees with work on an early HNB product by Pryor et al. ${ }^{32}$ and is consistent with work by Sussan et al. that found $7 \times 10^{11}$ longlived radicals/puff in TPM collected after 50 puffs, corresponding to 14 pmol for 12 puffs. ${ }^{43}$ The signal reported in the latter study is much narrower than the one that we found for the 3R4F cigarette (6.8 compared to $30 \mathrm{G}$ ), and no replicates are reported. In our hands, quantification of a signal of such low intensity is inaccurate. We further note that Gehling and Dellinger found a signal with a width of (6.49 \pm $1.69 \mathrm{G})$ from environmentally persistent free radicals in particulate matter $\mathrm{PM}_{2.5}$ extracted from air ${ }^{44}$ that shares the characteristic of the signal detected by Sussan et al. and at much higher level of 4.8 or $7.7 \mu \mathrm{mol}$ for eGO Vision and Blu ecigs by Lerner et al. ${ }^{45}$ Lerner et al. do not comment on their values, which are extremely large compared to values measured by others. Given their measurement parameters, for such a large amount of spins the signal-to-noise ratio should be by orders of magnitude higher than it is in the reported spectra. While it cannot be completely excluded that different e-cig devices produce very different levels of persistent radical in TPM, we can conclude that for the Solaris and MESH e-cigs as well as for the IQOS HNB product radical levels in TPM are below $7 \%$ of the level observed for 3R4F research cigarettes.

\section{CONCLUSION}

We have compared levels of free radicals and $\mathrm{NO}$ in the gas phase of aerosol as well as of persistent radicals in total particulate matter between the 3R4F research cigarette, two ecigs, and an HNB tobacco product by using spin-trapping techniques and EPR spectroscopy. We have applied standardized smoking and vaping regimes, reduced a number of factors that influence variability of the data, and have replicated our measurements. Signals from the research cigarette were found to be sufficiently strong for reliable quantification. For all vaping products, signals were of the same order of magnitude as the background signals, except for the NO level from the HNB tobacco product. The NO level in solutions of whole smoke increases with time for the research cigarette and remains constant for the HNB product. Considering this variation, the NO level of the HNB product is between 5.5 and $7.3 \%$ of that of the $3 \mathrm{R} 4 \mathrm{~F}$ cigarette.

Estimates of free radical level in the gas phase were obtained with $\mathrm{PBN}$ trapping in toluene and suggest that this level is reduced by $99 \%$ or more in the e-cigs and the HNB product compared to the $3 \mathrm{R} 4 \mathrm{~F}$ cigarette.
Similar amounts of TPM were found in all products, with the one for MESH being slightly higher and the ones for IQOS and Solaris being slightly lower than the one of 3R4F research cigarettes. However, only the TPM from the research cigarette contained persistent free radicals at a level that could be reliably distinguished from the background. Radical levels of the other products are below $7 \%$ of the level observed for the 3R4F cigarette.

Our findings demonstrate that free radicals levels are substantially reduced in e-cigarettes and heat-not-burn products compared to conventional cigarettes. A similar reduction of other HPHCs has been found before by other techniques. This supports the view that e-cigarettes and heatnot-burn products are a potentially less harmful alternative to cigarette smoking, although we maintain that toxicological studies are required to draw firm conclusions. We believe that our results can guide such studies and help in their interpretation.

\section{ASSOCIATED CONTENT}

\section{S Supporting Information}

The Supporting Information is available free of charge on the ACS Publications website at DOI: 10.1021/acs.chemrestox.9b00085.

Photos and schemes of the radical trapping and EPR measurement setup, supplemental figures on deoxygenation, trapping efficiency, trapping from whole smoke, particulate matter EPR spectrum, air blank spectrum, NO buildup kinetics, table on variability of BMPO trapping experiments, and detailed information on spin trap selection deoxygenation, and NO buildup kinetics (PDF)

\section{AUTHOR INFORMATION}

\section{Corresponding Author}

*E-mail: gjeschke@ethz.ch.

ORCID

Gunnar Jeschke: 0000-0001-6853-8585

\section{Present Address}

†Department of Chemistry, TU München, Lichtenbergstrasse 4, 85747 Garching, Germany

\section{Notes}

The authors declare no competing financial interest.

\section{ACKNOWLEDGMENTS}

The smoking machine, all smoking products, and financial assistance for buying consumables as well as publishing open access were kindly provided by Philip Morris, Inc. R\&D, Neuchâtel, Switzerland. We are grateful for helpful discussions on standard protocols and necessary controls to Arno Knorr, Falk Radtke, Mark C. Bentley, and Serge Maeder.

\section{ABBREVIATIONS}

BMPO, 5-tert-butoxycarbonyl-5-methyl-1-pyrroline- $\mathrm{N}$-oxide; CORESTA, Cooperation Centre for Scientific Research Relative to Tobacco; DEPMPO, 5-(diethoxyphosphoryl)-5methyl-1-pyrroline- $N$-oxide; DETC, diethyldithiocarbamate; DIPPMPO, 5-(diisopropoxyphosphoryl)-5-methyl-1-pyrroline- $\mathrm{N}$-oxide; DNA, desoxyribonucleic acid; DMPIO, 2,2dimethyl-4-phenyl-2H-imidazole-1-oxide; DMPO, 5,5-dimethyl-1-pyrroline- $\mathrm{N}$-oxide; e-cig, electronic cigarette; EPR, elec- 
tron paramagnetic resonance; FDA, U.S. Food and Drug Administration; HC, Health Canada; HNB, heat-not-burn; HPHCs, Harmful and Potentially Harmful Constituents; PBN, $N$-tert-butyl- $\alpha$-phenyl-nitrone; ROS, reactive oxygen species; TMIO, 2,2,4-trimethyl- $2 \mathrm{H}$-imidazole-1-oxide; TPM, total particulate matter; WHO, World Health Organization

\section{REFERENCES}

(1) WHO global report: mortality attributable to tobacco, 2012, WHO: Geneva, Switzerland.

(2) Harmful and Potentially Harmful Constituents in Tobacco Products and Tobacco Smoke: Established List. US Food and Drug Administration. Website https://www.fda.gov/tobaccoproducts/ guidancecomplianceregulatoryinformation/ucm297786.htm (accessed December 7, 2018).

(3) Notley, C., Ward, E., Dawkins, L., and Holland, R. (2018) The unique contribution of e-cigarettes for tobacco harm reduction in supporting smoking relapse prevention. Harm. Reduct. J. 15, 31 .

(4) Rodu, B. (2011) The scientific foundation for tobacco harm reduction, 2006-2011. Harm. Reduct. J. 8, 19.

(5) Stephens, W. E. (2018) Comparing the cancer potencies of emissions from vapourised nicotine products including e-cigarettes with those of tobacco smoke. Tob. Control 27, 10-17.

(6) National Academies of Sciences, Engineering, and Medicine, Public health consequences of e-cigarettes (2018) The National Academic Press, Washington DC.

(7) Canistro, D., Vivarelli, F., Cirillo, S., Babot Marquillas, C., Buschini, A., Lazzaretti, M., Marchi, L., Cardenia, V., RodriguezEstrada, M. T., Lodovici, M., Cipriani, C., Lorenzini, A., Croco, E., Marchionni, S., Franchi, P., Lucarini, M., Longo, V., Della Croce, C. M., Vornoli, A., Colacci, A., Vaccari, M., Sapone, A., and Paolini, M. (2017) E-cigarettes induce toxicological effects that can raise the cancer risk. Sci. Rep. 7, 2028.

(8) Mallock, N., Boss, L., Burk, R., Danziger, M., Welsch, T., Hahn, H., Trieu, H. L., Hahn, J., Pieper, E., Henkler-Stephani, F., Hutzler, C., and Luch, A. (2018) Levels of selected analytes in the emissions of "heat not burn" tobacco products that are relevant to assess human health risks. Arch. Toxicol. 92, 2145-2149.

(9) Lyons, M. J., Gibson, J. F., and Ingram, D. J. (1958) Free-radicals produced in cigarette smoke. Nature 181, 1003-4.

(10) Borish, E. T., Cosgrove, J. P., Church, D. F., Deutsch, W. A., and Pryor, W. A. (1985) Cigarette tar causes single-strand breaks in DNA. Biochem. Biophys. Res. Commun. 133, 780-6.

(11) Messner, B., and Bernhard, D. (2014) Smoking and cardiovascular disease: mechanisms of endothelial dysfunction and early atherogenesis. Arterioscler., Thromb., Vasc. Biol. 34, 509-15.

(12) Andersen, J. K., Davies, K. J., and Forman, H. J. (2013) Reactive oxygen and nitrogen species in neurodegeneration. Free Radical Biol. Med. 62, 1-3.

(13) Balbo, S., and Stepanov, I. (2018) The Wild West of ECigarettes. Chem. Res. Toxicol. 31, 823-824.

(14) Dellinger, B., Khachatryan, L., Masko, S., and Lomnicki, S. (2011) Free Radicals in Tobacco Smoke. Mini-Rev. Org. Chem. 8, 427-433.

(15) Robinson, E. A., and Johnson, J. D. (2011) Methods for Analysis of Free Radicals in Cigarette Smoke. Mini-Rev. Org. Chem. 8, 401-411.

(16) Kemp, T. J. (1999) Kinetic aspects of spin trapping. Prog. React. Kinet. Mech. 24, 287-358.

(17) Vanin, A. F. (1999) Iron diethyldithiocarbamate as spin trap for nitric oxide detection. Methods Enzymol. 301, 269-79.

(18) Burns, D. M., Dybing, E., Gray, N., Hecht, S., Anderson, C., Sanner, T., O'Connor, R., Djordjevic, M., Dresler, C., Hainaut, P., Jarvis, M., Opperhuizen, A., and Straif, K. (2008) Mandated lowering of toxicants in cigarette smoke: a description of the World Health Organization TobReg proposal. Tob. Control 17, 132-41.
(19) Tayyarah, R., and Long, G. A. (2014) Comparison of select analytes in aerosol from e-cigarettes with smoke from conventional cigarettes and with ambient air. Regul. Toxicol. Pharmacol. 70, 704-10.

(20) Roemer, E., Schramke, H., Weiler, H., Buettner, A., Kausche, S., Weber, S., Berges, A., Stueber, M., Muench, M., Trelles-Sticken, E., Pype, J., Kohlgrueber, K., Voelkel, H., and Wittke, S. (2012) Mainstream Smoke Chemistry and in Vitro and In Vivo Toxicity of the Reference Cigarettes 3R4F and 2R4F. Beiträge zur Tabakforschung International/Contributions to Tobacco Research 25, 316-335.

(21) Bitzer, Z. T., Goel, R., Reilly, S. M., Elias, R. J., Silakov, A., Foulds, J., Muscat, J., and Richie, J. P., Jr. (2018) Effect of flavoring chemicals on free radical formation in electronic cigarette aerosols. Free Radical Biol. Med. 120, 72-79.

(22) Goel, R., Durand, E., Trushin, N., Prokopczyk, B., Foulds, J., Elias, R. J., and Richie, J. P., Jr (2015) Highly reactive free radicals in electronic cigarette aerosols. Chem. Res. Toxicol. 28, 1675-7.

(23) Goel, R., Bitzer, Z., Reilly, S. M., Trushin, N., Foulds, J., Muscat, J., Liao, J., Elias, R. J., and Richie, J. P. (2017) Variation in Free Radical Yields from U.S. Marketed Cigarettes. Chem. Res. Toxicol. 30, $1038-1045$.

(24) Santos, J. H., Bond, A. M., Mocak, J., and Cardwell, T. J. (1994) Voltammetry of copper diethyldithiocarbamate in toluene and toluene-based solvents - development of a solvent extraction-stripping method for the determination of copper using microdisk electrodes. Anal. Chem. 66, 1925-1930.

(25) Baum, S. L., Anderson, I. G. M., Baker, R. R., Murphy, D. M., and Rowlands, C. C. (2003) Electron spin resonance and spin trap investigation of free radicals in cigarette smoke: development of a quantification procedure. Anal. Chim. Acta 481, 1-13.

(26) Stoll, S., and Schweiger, A. (2006) EasySpin, a comprehensive software package for spectral simulation and analysis in EPR. J. Magn. Reson. 178, 42-55.

(27) Davies, M. J., and Slater, T. F. (1986) Studies on the photolytic breakdown of hydroperoxides and peroxidized fatty acids by using electron-spin-resonance spectroscopy - spin trapping of alkoxyl and peroxyl radicals in organic solvents. Biochem. J. 240, 789-795.

(28) Stolze, K., Udilova, N., and Nohl, H. (2000) Spin trapping of lipid radicals with DEPMPO-derived spin traps: detection of superoxide, alkyl and alkoxyl radicals in aqueous and lipid phase. Free Radical Biol. Med. 29, 1005-14.

(29) Menzel, E. R., Vincent, W. R., and Wasson, J. R. (1976) Dipolar broadening of electron-spin resonance lines of free radicals - Spintrapping studies of free radicals in cigarette smoke. J. Magn. Reson. 21, $321-330$.

(30) Pryor, W. A., Terauchi, K., and Davis, W. H., Jr. (1976) Electron spin resonance (ESR) study of cigarette smoke by use of spin trapping techniques. Environ. Health Perspect. 16, 161-76.

(31) Margham, J., McAdam, K., Forster, M., Liu, C., Wright, C., Mariner, D., and Proctor, C. (2016) Chemical Composition of Aerosol from an E-Cigarette: A Quantitative Comparison with Cigarette Smoke. Chem. Res. Toxicol. 29, 1662-1678.

(32) Pryor, W. A., Church, D. F., Evans, M. D., Rice, W. Y., and Hayes, J. R. (1990) A comparison of the free radical chemistry of tobacco-burning cigarettes and cigarettes that only heat tobacco. Free Radical Biol. Med. 8, 275-9.

(33) Reszka, K., and Chignell, C. F. (1991) Spin-trapping of the superoxide radical in aprotic solvents. Free Radical Res. Commun. 14, 97-106.

(34) Reinke, L. A., Kotake, Y., McCay, P. B., and Janzen, E. G. (1991) Spin-trapping studies of hepatic free radicals formed following the acute administration of ethanol to rats: in vivo detection of 1 hydroxyethyl radicals with PBN. Free Radical Biol. Med. 11, 31-9.

(35) Astolfi, P., Greci, L., and Panagiotaki, M. (2005) Spin trapping of nitrogen dioxide and of radicals generated from nitrous acid. Free Radical Res. 39, 137-44.

(36) Shinagawa, K., Tokimoto, T., and Shirane, K. (1998) Spin trapping of nitric oxide in aqueous solutions of cigarette smoke. Biochem. Biophys. Res. Commun. 253, 99-103. 
(37) Counts, M. E., Morton, M. J., Laffoon, S. W., Cox, R. H., and Lipowicz, P. J. (2005) Smoke composition and predicting relationships for international commercial cigarettes smoked with three machine-smoking conditions. Regul. Toxicol. Pharmacol. 41, 185-227.

(38) Bartalis, J., Chan, W. G., and Wooten, J. B. (2007) A new look at radicals in cigarette smoke. Anal. Chem. 79, 5103-6.

(39) Bartalis, J., Zhao, Y. L., Flora, J. W., Paine, J. B., and Wooten, J. B. (2009) Carbon-centered radicals in cigarette smoke: acyl and alkylaminocarbonyl radicals. Anal. Chem. 81, 631-41.

(40) Dellinger, B., Pryor, W. A., Cueto, R., Squadrito, G. L., Hegde, V., and Deutsch, W. A. (2001) Role of free radicals in the toxicity of airborne fine particulate matter. Chem. Res. Toxicol. 14, 1371-7.

(41) Schaller, J. P., Pijnenburg, J. P., Ajithkumar, A., and Tricker, A. R. (2016) Evaluation of the Tobacco Heating System 2.2. Part 3: Influence of the tobacco blend on the formation of harmful and potentially harmful constituents of the Tobacco Heating System 2.2 aerosol. Regul. Toxicol. Pharmacol. 81, S48-S58.

(42) Gonzalez-Suarez, I., Martin, F., Marescotti, D., Guedj, E., Acali, S., Johne, S., Dulize, R., Baumer, K., Peric, D., Goedertier, D., Frentzel, S., Ivanov, N. V., Mathis, C., Hoeng, J., and Peitsch, M. C. (2016) In Vitro Systems Toxicology Assessment of a Candidate Modified Risk Tobacco Product Shows Reduced Toxicity Compared to That of a Conventional Cigarette. Chem. Res. Toxicol. 29, 3-18.

(43) Sussan, T. E., Gajghate, S., Thimmulappa, R. K., Ma, J., Kim, J. H., Sudini, K., Consolini, N., Cormier, S. A., Lomnicki, S., Hasan, F., Pekosz, A., and Biswal, S. (2015) Exposure to electronic cigarettes impairs pulmonary anti-bacterial and anti-viral defenses in a mouse model. PLoS One 10, No. e0116861.

(44) Gehling, W., and Dellinger, B. (2013) Environmentally persistent free radicals and their lifetimes in PM2.5. Environ. Sci. Technol. 47, 8172-8.

(45) Lerner, C. A., Sundar, I. K., Watson, R. M., Elder, A., Jones, R., Done, D., Kurtzman, R., Ossip, D. J., Robinson, R., McIntosh, S., and Rahman, I. (2015) Environmental health hazards of e-cigarettes and their components: Oxidants and copper in e-cigarette aerosols. Environ. Pollut. 198, 100-7. 